\title{
Mutational and functional analysis of the BVES gene coding region in Chinese patients with non-syndromic tetralogy of Fallot
}

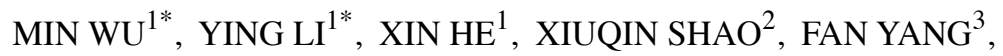 \\ MING ZHAO ${ }^{4}$, CHONG WU ${ }^{3}$, CUIFANG ZHANG ${ }^{3}$ and LEI ZHOU $^{1}$ \\ ${ }^{1}$ Department of Cardiology, The First Affiliated Hospital of Nanjing Medical University, Nanjing; \\ ${ }^{2}$ Department of Cardiology, Shangyu People's Hospital, Shangyu, Zhejiang; ${ }^{3}$ Department of Human Population Genetics, \\ Institute of Molecular Medicine, Peking University, Beijing; ${ }^{4}$ Department of Congenital Heart Disease, \\ General Hospital, Shenyang Military Area Command, Shenyang, P.R. China
}

Received November 16, 2012; Accepted December 21, 2012

DOI: $10.3892 / \mathrm{ijmm} .2013 .1275$

\begin{abstract}
Tetralogy of Fallot (TOF) is a common congenital heart defect (CHD). However, the genetic causes are largely unknown. Blood vessel epicardial substance (BVES) is postulated to play a role in embryonic development, and we previously found that the expression of BVES is upregulation in patients with congenital septal defect and that BVES participates in cardiocyte differentiation. We hypothesized that BVES is a candidate gene of TOF. In the present study, the entire coding sequence and splice junctions of BVES were sequenced in 114 unrelated patients with TOF and 400 unrelated healthy individuals used as controls. The functional effects of the exon mutant BVES were characterized in contrast to its wild-type counterpart using a luciferase reporter assay system. Four novel BVES mutations (c.166T $>$ C, c.909C $>$ T, c. $540-80 \mathrm{C}>\mathrm{T}, \mathrm{c} .958+30 \mathrm{~A}>\mathrm{G}$ ) were identified in patients with TOF but not in the 400 controls. Functional analysis indicated that the c.166T $>\mathrm{C}$ mutation of BVES was associated with an increased transcriptional activity. This finding suggests that BVES as a novel TOF gene may provide further insight into the molecular mechanisms involved in TOF.
\end{abstract}

\section{Introduction}

Congenital heart defects (CHDs) are the most common developmental abnormalities with a prevalence of $\sim 1 \%$ in neonates, and are the leading non-infectious cause of infant mortality, accounting for $>29 \%$ of infants who die from a birth defect $(1,2)$. TOF is a common CHD characterized by a gross

Correspondence to: Dr Lei Zhou, Department of Cardiology, The First Affiliated Hospital of Nanjing Medical University, 300 Guangzhou Road, Nanjing, Jiangsu 210029, P.R. China

E-mail: zhoulei7005@hotmail.com

*Contributed equally

Key words: BVES gene, tetralogy of Fallot, variants, congenital heart defects, heart development structural abnormality of the heart with functional significance (3) and is complicated by ventricular septal defects, atrial septal defects or abnormalities in the branching pattern of coronary arteries, obstruction to right ventricular outflow tract (RVOT), aortic dextroposition (AD) and right ventricular hypertrophy (RVH). The abnormality of TOF starts during the first eight weeks of fetal growth and affects $\sim 1$ in 3,000 live newborns (4). Clinical symptoms include cyanosis/clubbing, hypoxia, breathlessness, refusal to feed, failure to gain weight and severe congenital heart malformation.

Classic TOF and its variants have been observed as a heritable syndrome such as Alagille syndrome and Di Georges syndrome. Prenatal infections, exposure to teratogens, maternal illness and folate deficiency are the few known causes. However, $70 \%$ of TOF cases also occur sporadically, without any other anomaly, and from unknown causes (5). The cellular and molecular mechanisms underlying TOF are complex and involve cell coordinated growth, specification, differentiation, migration and apoptosis, morphogenesis and cell-cell interaction (6), and are poorly understood and difficult to study in humans. To date, a few genes which cause TOF have been identified. Mutations in NKX2.5 (7), GATA4 (8), TBX5 (9) and others are associated with TOF. However, similar to most CHDs, TOF is thought to be a multigenic disorder, and the basic mechanisms of TOF in humans are still incompletely defined. We aimed to identify the associated causal genes of TOF and study the pathogenesis of these genes in order to reduce the incidence of CHDs, improving the quality of life of this population and lowering the perinatal mortality rate.

Blood vessel epicardial substance (BVES, also known as Popdic1 or Pop1) was discovered in 1999 by two independent laboratories using screening to identify novel genes that were highly expressed in the developing heart $(10,11)$. As an evolutionarily conserved transmembrane protein, BVES has been postulated to play a role in cell signaling as it is localized at sites of intercellular contacts, bound to the signaling modifier guanine nucleotide exchange factor $\mathrm{T}$, and has been shown to be involved in Rho signaling and receptor cycling (12). Moreover, BVES has been proposed to play a role in cell adhesion, epithelial integrity and cell motility (13-16), three cellular functions that are essential in embryonic gastrulation. Many 
studies have demonstrated that BVES plays an important role in heart development, heart failure and arrhythmia $(17,18)$. Previously, we identified differentially expressed genes between ventricular septal defect (VSD) and normal ventricular septum myocardium using suppression subtractive hybridization. Then, among these differentially expressed genes, we found that the BVES gene was upregulated in the ventricular septum of VSD patients by bioinformatics analysis (19). We also found that Bves expression was increased 2-fold in cardiocyte differentiation prophase in an induced p19cl6 differentiation model. Therefore, we hypothesized that Bves plays an important role in cardiac development. To date, there are no reports that the BVES gene exhibits mutations in patients with CHDs.

Herein, we sequenced the entire coding region and splice junctions of BVES in 114 unrelated patients with TOF. The functional characteristics of the mutant BVES were analyzed using a luciferase reporter assay system.

\section{Materials and methods}

Samples. In the present study, blood samples were obtained from 114 patients with TOF and 114 matched individuals with no reported cardiac phenotype as controls who were patients hospitalized at two medical centers in Nanjing City and Shenyang City, respectively. In our study, the patients were diagnosed according to their past histories, physical examination, 12-lead electrocardiograms, and ultrasonic echocardiogram; most of the patients had cardiac catheterization examination data and/or operative reports. To assess the allelic frequency, another 400 unrelated healthy individuals from Peking University Hospital received annual routine health surveys during the same period. Patients with other congenital or acquired heart diseases were excluded from this study. Samples of peripheral blood (2-3 ml) were collected from all participants after ethical approval by the Ethics Committee of Nanjing Medical University. Written informed consent was obtained from all participants or their parents in this human population study which conformed to the principles outlined in the Declaration of Helsinki.

Sequencing and analysis of the purified DNA. Human genomic DNA was isolated from EDTA-anticoagulated blood using the proteinase $\mathrm{K}$ methods as previously described (20). DNA fragments of 200-400 bp were amplified by PCR from $10 \mathrm{ng}$ of genomic DNA from each participant using the primers listed in Table I. The amplified DNA fragments were purified by PEG precipitation and subjected to direct sequencing on ABI 3130XL according to the manual description of BigDye v3.1. The gene has three transcripts, all of them coding 360 amino acid. BVES transcript 2 that represents the variant $\mathrm{A}$ and variants $B$ and $C$ encode the same protein. We sequenced the coding sequence (from exon 2 to exon 8) of BVES transcript 2 (NM_007073, gil313760594I). Sequencing results were analyzed by SeqScanner v1.0 (Applied Biosystems) and Mutation Surveyor v3.10 (Soft Genetics) against references [GenBank sequence (NG_016625.1) and sequences from controls in this study].

Plasmid construction and cell transfection. Total RNA was isolated from the hearts of the patients with hypertrophic cardiomyopathy using TRIzol reagent (Invitrogen) according to the manufacturer's instructions. The obtained cDNA was subjected to PCR amplification of a human Bves encoding region with the primers: 5'-CTCCTCGAGGGCAAGCCCCT TGGAATTTT-3' and 5'-TCTAAGCTTAAGGCAGCTGA TGGACTTTC-3'. The PCR was carried out using Pfu polymerase (Tianlab) with the following cycle profile: $3 \mathrm{~min}$ at $95^{\circ} \mathrm{C}$ followed by 35 cycles of $95^{\circ} \mathrm{C}$ for $30 \mathrm{sec}, 55^{\circ} \mathrm{C}$ for $45 \mathrm{sec}$ and $72^{\circ} \mathrm{C}$ for $60 \mathrm{sec}$. The resulting PCR products were then digested by XhoI and HindIII and ligated into the pGL3-promoter vector according to the technical manual (Promega Corp., Madison, WI, USA) forming plasmids pGL3-Bves-WT. To create the c.166T $>C$ and the c.909C $>$ T mutants, single-nucleotide point mutations were introduced by site-directed mutagenesis using overlap extension PCR technique. Wild-type Bves in the pGL3CMV-luciferase vector was used as the template forming pGL3-exon2/7-mt. The accuracy of the plasmids was confirmed by DNA sequencing.

We constructed plasmids that carried a CMV (human cytomegalovirus promoter region) promoter, the human full length coding region of BVES, and Firefly luciferase fused at its 3 '-end. The translational efficiency was measured by Firefly luciferase activity. Transfection efficiency was standardized by reference to Renilla luciferase activity resulting from the parallel introduction of this plasmid into the test cells.

HEK293A cells (60-80\% confluence) in 24-well plates were transiently transfected with $0.66 \mu \mathrm{g}$ of Bves-Firefly luciferase fusion plasmid and $0.33 \mu \mathrm{g}$ of PRL-TK reference Renilla luciferase plasmid (Promega Corp.) using VigoFect (Vigonous) according to the manufacturer's instructions. Forty-eight hours after transfection, cells were harvested and luciferase activity (Firefly and Renilla) was measured using a Dual-Luciferase Reporter Assay kit (Promega Corp.) and a multi-well fluorometer (BioTek, USA). The protein expression level of the Bves gene was measured by the ratio of Firefly to Renilla. Data represent the means \pm SEM of twelve independent experiments carried out in triplicate.

Statistical analysis. Sets of data in the various groups were compared using the unpaired two-tailed t-test and expressed as the means \pm SD. A p-value of $<0.05$ was considered to indicate a statistically significant result.

\section{Results}

Identification of genetic variants in BVES. The entire Bves coding sequences of all the subjects were analyzed and compared with the GeneBank human Bves coding sequence. We identified four novel varations (c.166T $>$ C p.L56L, c. 909 C $>$ T p.D303D, c. $540-80 G>A$, c. $958+30 A>G)$ in four unrelated patients with TOF which were not noted in the 114 controls nor in the additional 400 controls (Fig. 1). In addition, we replicated six reported single-nucleotide polymorphisms (SNPs) (Table II). No significant differences were found in the allelic frequencies of these SNPs between the TOF patients and normal controls. Specifically, variant c.166T $>C$ at the three transmembrane helices of Bves predicted that Bves may be lost at the cell surface. A heterozygous (c.909C $>$ T p.D303D) and a homozygous (c.166T>C p.L56L) variant did not result in amino acid changes, but they may have been impacted by 
Table I. Sequences of primer pairs for DNA amplification and mutational analysis.

\begin{tabular}{lllcc}
\hline Exon & \multicolumn{1}{c}{ Forward primer $\left(5^{\prime}-3^{\prime}\right)$} & \multicolumn{1}{c}{ Reverse primer $\left(5^{\prime}-3^{\prime}\right)$} & Length $(\mathrm{bp})$ & Annealing temperature $\left({ }^{\circ} \mathrm{C}\right)$ \\
\hline E2-1 & GAATCTGGGTCTGTCAAT & GGCACAGGTATGATACTT & 232 & 55 \\
E2-2 & GAATCAACTGCCATAGGT & CTCTGAAGCTCTCAATGTA & 273 & 50 \\
E3-1 & CTGCCTGAGATGTGAATC & CACAGAGTTCCAGATCAT & 350 & 55 \\
E3-2 & CCGATGTGCCTTGGATAT & CAGAAAGCCTAAACTTCAG & 226 & 50 \\
E4 & GCTGCATTGGTGAAGCAT & CTCCATTCATTGGCAACAT & 327 & 50 \\
E5 & GTCCATCATCTCTGTGGAAT & CCTTTGGATTCTGAATGAGA & 301 & 55 \\
E6-1 & GTCCACTAAATCATTCCTAG & GTGGGATCATTCAATGAGT & 249 & 54 \\
E6-2 & GTCCACTAAATCATTCCTAG & GTGGGATCATTCAATGAGT & 279 & 55 \\
E7 & GGAATGTATGATTCAACTCC & CCAGAGATACCCATCAGA & 250 & 55 \\
E8-1 & GGTGCTGCTAGTGATAGAT & GGATCTCTTCAAGACACCTT & 297 & 55 \\
E8-2 & GTCCATCAGCTGCCTTGAT & GCATAGTCAGAAGGCTCAGT & 242 & 55 \\
E8-3 & GGTGTCTTGAAGAGATCCT & GGACAACCTCATTGCTAT & 356 & 55 \\
\end{tabular}

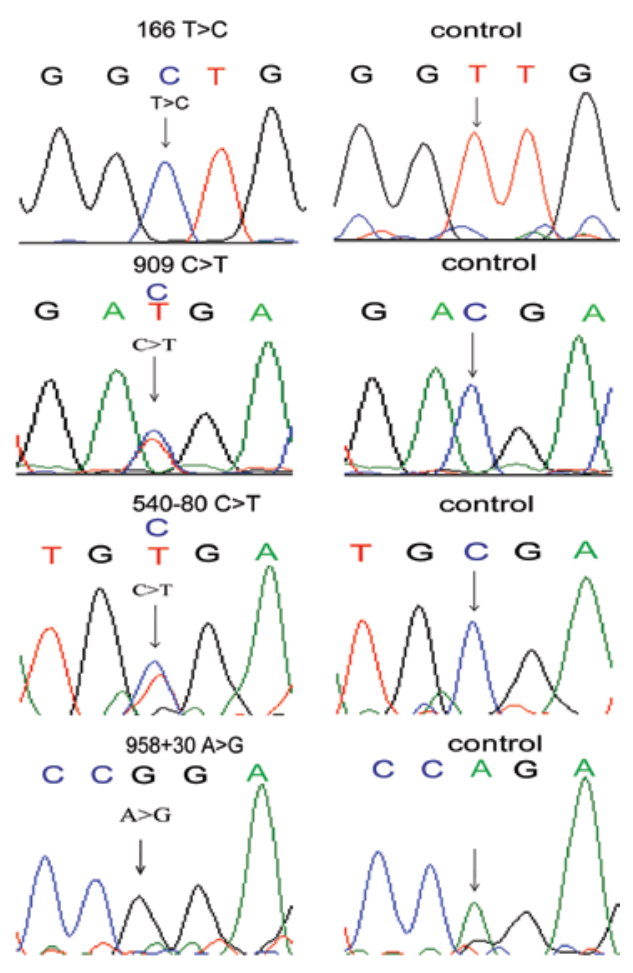

Figure 1. Genetic variants of BVES screened in this study. Corresponding nucleotides in the controls T, C, C and A, respectively, are presented in the upper panel.

the nucleotide splice or the stability of RNA. We analyzed the nucleotide conservation of the two variants and found that the c.166T $>C$ variant was evolutionarily conserved (Fig. 2). To ascertain the mutations, known TOF genes (i.e., GATA4, Nkx2.5, TBX5 and FOG2) were sequenced and excluded from the BVES variant carriers. Clinic diagnosis of four carriers was TOF with no clinical manifestations of cardiomyopathies or other self-reported inherited diseases. It was not known whether other family members of the BVES mutation carriers had the same mutation due to a failure to obtain a signed consent form.

\begin{tabular}{|c|c|}
\hline & c. $166 \mathrm{~T}>\mathrm{C}$ \\
\hline Homo sapiens & TGCAGTTGGG T|TGGTTATTCCAACT \\
\hline Pan troglodytes & TGCAGTTGGG|TGGTTATTCCAACT \\
\hline Gorilla gorilla & TGCAGTTGGG TTGGTTATTCCAACT \\
\hline Pongo abelii & TGCAGTTGGGTTGGTTATTCCAACT \\
\hline Macaca mulatta & TGCAGTTGGGT|TGGTTATTCCAACT \\
\hline callithrix jacchus & TGCAGTTGGGTTGATTATTCCAACT \\
\hline Mus musculus & TGCAGTTGGGT|TGATTATTCCAACT \\
\hline Rattus norvegicus & \\
\hline Bos taurus & CGCAATTGGATTTGGTTATTCCAACT \\
\hline Sus scrofa & TGCCATTGGG TTGGTTCTTCCAACT \\
\hline Canis familiaris & CACAATTGGG TTGGTTATTCCAACT \\
\hline Equus caballus & TGCCATTGGTITGATTATTCCAACT \\
\hline & c. $909 \mathrm{C}>\mathrm{T}$ \\
\hline Homo sapiens & CAGTGACAGTGA CACGGCTTGCA \\
\hline Pan troglodytes & CAGTGACAGTGA CACGGCTTGCA \\
\hline Gorilla gorilla & CAGTGACAGTGA C GACGGCTTGCA \\
\hline Pongo abelii & CAGTGACAGTGA C GACGGCTTGCA \\
\hline Macaca mulatta & CAGTGACAGTGA CACGGCTTGCA \\
\hline callithrix jacchus & CAGTGACAGTGA CAGGGCTTGCA \\
\hline Mus musculus & CAGCGACGGCGAG GACGGCCTGCA \\
\hline Rattus norvegicus & …………...... \\
\hline Bos taurus & AGCGAAGACGAA|GAGGCTTACA \\
\hline Sus scrofa & AGTGACAGCGAG GACGGCTTACA \\
\hline Canis familiaris & AGTGACAGTGAT GACGGCTTACA \\
\hline Equus caballus & AGTGACAGCGAA GACGGCTTACA \\
\hline
\end{tabular}

Figure 2. Multiple sequence alignment of BVES nucleotides in different species. Sequence alignment showing the phylogenetic conservation of thymine (c.166T $>$ C) and cytosine (c.909C $>$ T) in the BVES nucleotide.

Functional implications. The potential functional effects of these variants were evaluated by bioinformatics analysis (Table III). It was found that c.166T $>C$ and c.958+30A $>\mathrm{G}$ potentially affected RNA splicing, while c.958+30A $>$ G produced a new silencer motif. The variant c.540-80C $>\mathrm{T}$ emerged as a new enhancer motif and c.909C $>$ T may not be affected by RNA splicing. 
Table II. Reported SNPs of the BVES gene in this study.

\begin{tabular}{|c|c|c|c|c|c|c|}
\hline Gene & Exon & Nucleotide & Protein & rs number & MAF & MAF reported \\
\hline BVES & 3 & c. $227-23 \mathrm{G}>\mathrm{A}$ & & rs4946656 & $\mathrm{G}=14 / 236=0.059$ & $\mathrm{C}=0.05$ (90 AoD Chinese $)$ \\
\hline BVES & 3 & c. $351+30 \mathrm{~T}>\mathrm{G}$ & & rs9404604 & $\mathrm{T}=17 / 124=0.137$ & $\mathrm{~A}=0.125(120 \mathrm{CHB}+\mathrm{JPT})$ \\
\hline BVES & 3 & c. $351+55 \mathrm{G}>\mathrm{A}$ & & rs72932419 & $A=15 / 168=0.089$ & $\mathrm{~T}=0.045(88 \mathrm{CHB}+\mathrm{JPT})$ \\
\hline BVES & 3 & c. $351+82 \mathrm{~T}>\mathrm{C}$ & & rs9404603 & $\mathrm{T}=23 / 168=0.137$ & $\mathrm{~A}=0.080(88 \mathrm{CHB}+\mathrm{JPT})$ \\
\hline BVES & 4 & c. $385 \mathrm{C}>\mathrm{T}$ & p.R129W & rs2275289 & $\mathrm{T}=4 / 228=0.0175$ & $\mathrm{~A}=0.070(86 \mathrm{HCB}) ; 0.037(82 \mathrm{CHB})$ \\
\hline BVES & 8 & c. ${ }^{*} 114 \mathrm{~A}>\mathrm{G}$ & & rs 221657 & $\mathrm{G}=26 / 232=0.112$ & $\mathrm{C}=0.098(86 \mathrm{HCB}) ; 0.081(82 \mathrm{CHB})$ \\
\hline
\end{tabular}

MAF, minor allele frequency.

Table III. Functional predictions of the variants detected in this study.

\begin{tabular}{lllll}
\hline Nucleotide change & Gene region & \multicolumn{1}{c}{ Wild-type } & \multicolumn{1}{c}{ Mutant } & \multicolumn{1}{c}{ Predicted function } \\
\hline c.166T $>\mathrm{C}$ & Exon 2 & TGGGTTGGT & TGGGCTGGT & Splicing activity decreased by $-33.63 \%$ \\
c.909C $>\mathrm{T}$ & Exon 7 & ACAGTGACG & ACAGTGATG & Splicing activity decreased by $-0.44 \%$ \\
c.540-80C $>\mathrm{T} / \mathrm{G}>\mathrm{A}$ & Intron 4 & AAATCG & AAATCA & New site: enhancer motif \\
c.958+30A $>\mathrm{G}$ & Intron 7 & ATACTACTCCAGAG & ATACTACTCCGGAG & Splicing activity decreased by $-33.91 \%$ \\
& & AGAGTT & GGAGTT & New site: silencer motif \\
\hline
\end{tabular}

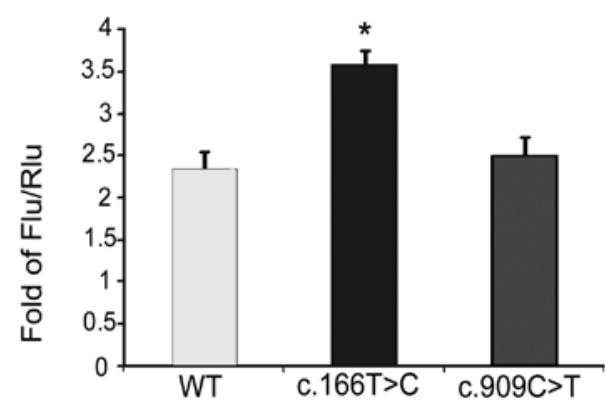

Figure 3. c.166T $>$ C increased protein production or transcriptional activity as determined by luciferase analysis. Flu, Firefly luciferase; Rlu, Renilla luciferase. ${ }^{*} \mathrm{P}<0.05$ vs. wild-type BVES.

Luciferase assay. To characterize the function of the mutations in exons of BVES, mutated sequences were cloned into plasmids and experiments were carried out compared with the wild-type control. It was found that $\mathrm{c} .166 \mathrm{~T}>\mathrm{C}$ increased the luciferase activity by 0.5 -fold (Fig. 3). Thus, we demonstrated that the bves mutation (c.166T $>$ C) found in TOF patients was gain-of-function.

\section{Discussion}

The most important finding in this study was the identification of four novel genetic variants within the BVES gene from the peripheral blood cells of TOF patients that were not noted in the 114 controls nor in the additional 400 controls. Bioinformatics analysis implied that these variants potentially affected motifbinding sites and splicing. We also demonstrated that the bves mutation (c.166T $>C$ ) found in TOF patients increased the luciferase activity by 0.5 -fold which was gain-of-function.
There was no significant difference in the luciferase activity between the variant c. $909 \mathrm{C}>\mathrm{T}$ and wild-type, thus it was likely a rare SNP.

Tetralogy of Fallot is the most common cyanotic congenital cardiac defect; it involves abnormalities in myocardialization resulting in a failure to muscularize the developing proximal outlet septum (21). TOF is of great importance to the pediatrician and cardiac surgeon since patients with these problems may require urgent and complicated surgery shortly after birth and long-term follow-up is needed (5). Understanding the aetiology of these defects would be useful for designing strategies for their prevention, either by allowing prenatal diagnosis or by therapeutic approaches such as vitamin supplementation that might reduce their incidence (22).

Embryonic cardiovascular development in humans is regulated by a diverse array of molecular signaling pathways that are activated by various intrinsic programs, molecular and morphogenic events (23). The morphogenesis of the human heart is a complex process, in which numerous genes are involved. BVES may play an important role in the signaling pathway for heart development.

BVES is a transmembrane protein which has also been considered as belonging to the cytoskeleton. These proteins play a role in the link with the extracellular matrix (24). The extracellular matrix that constitutes the cardiac jelly within the cushions has long been thought to play an important role in early septal morphogenesis (25). Abnormalities of the extracellular matrix cause atrioventricular canal and outlet septum defects (26). The differential expression of BVES in the heart of TOF patients may cause extracellular matrix disorder and may be involved in the development of TOF. Our previous study found that the cytoskeleton protein BVES was upregulated in septal defect patients (19). This study also found that 
the mutation c.166T $>C$ increased the transcription activity of BVES. Numerous studies have demonstrated that BVES plays an important role in heart and vascular development (27), heart failure (18) and arrhythmia (17). However, the present study was the first to demonstrate that BVES is involved in CHD, and may be a candidate gene of TOF.

Given the severe phenotypes noted in both $X$. laevis and D. melanogastor embryos when BVES is depleted, it was predicted that Bves-null mice would exhibit obvious developmental defects and would not live a normal lifespan (28). This, however, was not the case. Bves-null mice displayed no overt morphological defects. As the Popeye domain is highly conserved throughout all Popdc family members, and all three members have similar tissue expression, it is possible that Bves, Popdc2 and Popdc3 have redundant functions in development (29). It was demonstrated that mice lacking either Popdc1 or Popdc2 (also known as Pop2) are normal when housed under standard conditions but develop a striking agedependent sinus node dysfunction when subjected to physical or mental stress. However, Popdc1 and Popdc3 expression was not elevated in Popdc2 null mutants (17). Therefore, BVES, Popdc2 and Popdc3 do not have redundant functions in development. Previously, in differentially expressed genes, we found that the BVES gene was upregulated in the ventricular septum of VSD patients by bioinformatics analysis (19).

In conclusion, we demonstrated that the BVES gene exhibit mutations in patients with CHD, which were not previously identified. We performed a systematic screening for genetic mutations of the BVES gene in the peripheral blood cells of patients with TOF and identified four novel genetic variants in TOF cardiac tissues that were not found in the 114 controls and the additional 400 controls. These genetic variants may be associated with TOF through multiple mechanisms.

\section{Acknowledgements}

We thank all of the participants in our study population. This research was supported by the National Natural Science Fund of China (no. 30871079), the National Science Foundation of Jiangsu (no. BK2011770) and the Medical Key Talent Program of Jiangsu (no. K201110).

\section{References}

1. Roger VL, Go AS, Lloyd-Jones DM, et al: American Heart Association Statistics Committee and Stroke Statistics Subcommittee: Heart disease and stroke statistics - 2011 update: a report from the American Heart Association. Circulation 123: e18-e209, 2011.

2. Hoffman JI: Incidence of congenital heart disease: II. Prenata incidence. Pediatr Cardiol 16: 155-165, 1995.

3. Mitchell SC, Korones SB, Berendes HW, et al: Congenital heart disease in 56,109 births. Incidence and natural history. Circulation 43: 323-332, 1971.

4. Ferencz C, Rubin JD, McCarter RJ, et al: Congenital heart disease: prevalence in live birth. The Baltimore-Washington Infant Study. Am J Epidemiol 121: 31-36, 1981.
5. Kola S, Koneti NR, Golla JP, et al: Mutational analysis of JAG1 gene in non-syndromic Tetralogy of Fallot children. Clin Chim Acta 412: 2232-2236, 2011.

6. Kaynak B, von Heydebreck A, Mebus S, et al: Genome-wide array analysis of normal and malformed human hearts. Circulation 107: 2467-2474, 2003

7. Schott JJ, Benson DW, Basson CT, et al: Congenital heart disease caused by mutations in the transcription factor NKX2-5. Science 281: 108-111, 1998.

8. Okubo A, Miyoshi O, Baba K, Takagi M, et al: A novel GATA4 mutation completely segregated with atrial septal defect in a large Japanese family. J Med Genet 41: e97, 2004.

9. Basson CT, Huang T, Lin RC, et al: Different TBX5 interactions in heart and limb defined by Holt-Oram syndrome mutations. Proc Natl Acad Sci USA 96: 2919-2924, 1999.

10. Reese DE, Zavaljevski M, Streiff NLand Bader D: bves: a novel gene expressed during coronary blood vessel development. Dev Biol 209: 159-171, 1999.

11. Andree B, Hillemann T, Kessler-Icekson G, et al: Isolation and characterization of the novel popeye gene family expressed in skeletal muscle and heart. Dev Biol 223: 371-382, 2000.

12. Russ PK, Kupperman AI, Presley SH, et al: Inhibition of RhoA signaling with increased Bves in trabecular meshwork cells. Invest Ophthalmol Vis Sci 51: 223-230, 2010.

13. Wilson $P$ and Keller R: Cell rearrangement during gastrulation of Xenopus: direct observation of cultured explants. Development 112: 289-300, 1991.

14. Russ PK, Pino CJ, Williams CS, et al: Bves modulates tight junction associated signaling. PLoS One 6: e14563, 2011.

15. Hager HA and Bader DM: Bves: ten years after. Histol Histopathol 24: 777-787, 2009.

16. Hager HA, Roberts RJ, Cross EE, et al: Identification of a novel Bves function: regulation of vesicular transport. EMBO J 29: 532-545, 2010.

17. Boukens BJ and Christoffels VM: Popeye proteins: muscle for the aging sinus node. J Clin Invest 122: 810-813, 2012.

18. Gingold-Belfer R, Bergman M, Alcalay Y, et al: Popeye domaincontaining 1 is down-regulated in failing human hearts. Int J Mol Med 27: 25-31, 2011

19. Zhang H, Zhou L, Yang R, et al: Identification of differentially expressed genes in human heart with ventricular septal defect using suppression subtractive hybridization. Biochem Biophys Res Commun 342: 135-144, 2006.

20. Tian XL and Wang QK: Generation of transgenic mice for cardiovascular research. Methods Mol Med 129: 69-81, 2006

21. Warnes CA: The adult with congenital heart disease: born to be bad? J Am Coll Cardiol: 46: 1-8, 2005.

22. Giannopoulos NM, Chatzis AC, Bobos DP, et al: Tetralogy of Fallot: influence of right ventricular outflow tract reconstruction on late outcome. Int J Cardiol 97 (Suppl 1): 87-90, 2004.

23. Jain R, Rentschler S and Epstein JA: Notch and cardiac outflow tract development. Ann NY Acad Sci 1188: 184-190, 2010.

24. Hein S, Kostin S, Heling A, et al: The role of the cytoskeleton in heart failure. Cardiovasc Res 45: 273-278, 2000.

25. Maslen CL: Molecular genetics of atrioventricular septal defects. Curr Opin Cardiol 19: 205-210, 2004.

26. Marino B and Digilio MC: Congenital heart disease and genetic syndromes: specific correlation between cardiac phenotype and genotype. Cardiovasc Pathol 9: 303-315, 2000.

27. Wada AM, Reese DE and Bader DM: Bves: prototype of a new class of cell adhesion molecules expressed during coronary artery development. Development 128: 2085-2093, 2001.

28. Andree B, Fleige A, Arnold HH, et al: Mouse Pop1 is required for muscle regeneration in adult skeletal muscle. Mol Cell Biol 22: 1504-1512, 2002.

29. Parnes D, Jacoby V, Sharabi A, et al: The Popdc gene family in the rat: molecular cloning, characterization and expression analysis in the heart and cultured cardiomyocytes. Biochim Biophys Acta 1769: 586-592, 2007. 